\title{
Clinical Efficacy of a New Robot-assisted Gait Training System for Acute Stroke Patients
}

\author{
Andy Chien ${ }^{1,2,3} \cdot$ Fei-Chun Chang ${ }^{2} \cdot$ Nai-Hsin Meng ${ }^{2} \cdot$ Pei-Yu Yang ${ }^{2} \cdot$ Ching Huang $^{1} \cdot$ Li-Wei Chou $^{1,2,4}$ (I)
}

Received: 15 September 2020 / Accepted: 10 December 2020 / Published online: 7 January 2021

(c) The Author(s) 2021

\begin{abstract}
Purpose Robot-assisted gait rehabilitation has been proposed as a plausible supplementary rehabilitation strategy in stroke rehabilitation in the last decade. However, its exact benefit over traditional rehabilitation remain sparse and unclear. It is therefore the purpose of the current study to comparatively investigate the clinical benefits of the additional robot-assisted training in acute stroke patients compared to standard hospital rehabilitation alone.

Methods Ninety acute stroke patients ( $<3$ month) were recruited. All participants received the standard hospital neurorehabilitation comprises 45-60 min sessions daily for 3 weeks. Sixty patients also received an additional 30 min of robot-assisted gait training with the HIWIN MRG-P100 gait training system after each of the standard neurorehabilitation session. Outcome measures included: 1. Berg Balance Scale (BBS); 2. Brunnstrom Stage; 3. Pittsburgh Sleep Quality Index and 4. Taiwanese Depression Questionnaire (TDQ) which were assessed pre-treatment and then after every five training sessions.

Results Both groups demonstrated significant improvement pre- and post-treatment for the BBS (robotic group $\mathrm{p}=0.023$; control group $p=0.033)$ but no significant difference $(p>0.1)$ between the groups were found. However, the robotic training group had more participants demonstrating larger BBS points of improvement as well as greater Brunnstrom stage of improvement, when compared to the control group. No significant within and between group statistical differences $(p>0.3)$ were found for Pittsburgh Sleep Quality Index and Taiwanese Depression Questionnaire.

Conclusion The addition of robotic gait training on top of standard hospital neurorehabilitation for acute stroke patients appear to produce a slightly greater improvement in clinical functional outcomes, which is not transferred to psychological status.
\end{abstract}

Keywords Stroke $\cdot$ Gait $\cdot$ Rehabilitation $\cdot$ Robotics $\cdot$ Psychological

\section{Introduction}

With the rapid transit into an aging society for most developed countries worldwide, stroke is quickly becoming the leading cause of long term functional disability globally [1].

Li-Wei Chou

chouliwe@gmail.com

1 Department of Physical Therapy, Graduate Institute of Rehabilitation Science, China Medical University, Taichung, Taiwan

2 Department of Physical Medicine and Rehabilitation, China Medical University Hospital, Taichung, Taiwan

3 Research and Development Center for Medical Devices, National Taiwan University, Taipei, Taiwan

4 Department of Physical Medicine and Rehabilitation, Asia University Hospital, Asia University, Taichung, Taiwan
Stroke associated disabilities is consequently becoming an enormous health and social economic burden on the public health system [2]. The types and degrees of disability the patient suffers following a stroke is largely depended upon which areas of the brain is affected, however, it has been reported that close to one-third of the surviving patients from stroke fail to regain independent walking ability and with most patients demonstrating some form of gait abnormality clinically even 5 years post-stroke [3]. It is therefore not surprising that addressing decreased ambulatory capacity is one of the key goals in modern rehabilitation. This is particularly important given the potential link between the loss of ambulatory capacity and living independence that can lead to psychosocial implications such as poorer quality of life, difficulties with activities of daily living and subsequently the development of depression in a portion of 
stroke survivors that failed to regain ambulatory independence $[4,5]$.

Despite the proven usefulness of therapist-assisted gait rehabilitation, with the ever-increasing strain and limited availability of health resources in the modern clinical setting, therapist-assisted gait rehabilitation is becoming less and less feasible as it is not only very labor-intensive but also resources demanding. With the advancement of technology and manufacturing techniques, electromechanicalassisted gait rehabilitation has received intensive interest in the last decade as a plausible supplementary rehabilitation technique in stroke rehabilitation [6, 7]. More specifically, as robotics are able to provide consistent repeatability as well as intensity adjustable training with relatively low demand on human resources, a recent Cochrane review concluded that electromechanical gait training devices are able to assist patients in achieving independence in walking or at least improve key walking parameters [8]. This was particularly true in acute stroke patients that is non-ambulatory as this sub-population of the stroke survivors provide the best opportunity for the recovery of lower limb motor function through the synthesis of neuroplasticity when provided with standardized and highly repetitive lower limb motor training [9].

Currently, despite the recent advances in the development and design of better robotics, the exact benefit of the robot-assisted therapy over traditional rehabilitation remain sparse and unclear [10]. More specifically, some research has reported results in favour of the conventional therapistassisted training [11, 12], other research yielded results in favour of robot-assisted training [13, 14], with others finding no significant difference between the two types of therapy [15-17]. Such inconsistent and contrasting results has been proposed to be due to the clinical heterogeneity of stroke and is the main obstacle for the wider clinical adaptation of robot-assisted gait rehabilitation [18].

It is therefore the purpose of the current study to comparatively investigate the clinical efficacy of a recently developed HIWIN Robotic Gait Training System (MRG-P100) in an acute stroke population and compare it with the traditional hospital-based rehabilitation programs. The MRGP100 system incorporates commonly used clinical "tilt table" design combined with an electromechanical-assisted system that allow patients to receive weight-bearing gait re-education training without a suspension system, which is more cost effective compared with other commercially available devices. Acute stroke patients were chosen in order to minimize the heterogeneity of the sample patients that is also most likely to benefit from intense robotic training. We aim to provide objective measurements of the time-course of changes in lower limb motor function as well as the potential psychological benefits of prescribing robotic-assisted gait training for acute stroke survivors.

\section{Materials and Methods}

\subsection{Patient Population}

In this study, acute stroke patients were recruited between June 2016 and May 2018 from the Department of Physical Medicine and Rehabilitation at China Medical University Hospital. The inclusion criteria were: (1) age between 20 and 80-year old; (2) 1st-ever single unilateral corticalsubcortical stroke confirmed by brain imaging that is either ischemic or haemorrhagic; (3) onset of stroke to be less than 3 months [19] and is unable to ambulate independently including the use of any assistive device; (4) able to walk independently prior to stroke and (5) able to comprehend and cooperate with the evaluation procedure. The exclusion criteria were: (1) patients deemed by a physician to be medically unstable; (2) cognitive impairments that would impact on the safe participation in the study (MMSE <23); (3) other prior musculoskeletal conditions that affected gait capacity; (4) co-existence of other neurological diseases and (5) participants receiving additional treatments that is outside the scope of standardized hospital rehabilitation program as per the study protocol.

All participants or appropriate legal carer gave their informed consent and the Institution's ethical committee granted the ethical approval for the study.

\subsection{HIWIN Robotic Gait Training System (MRG-P100)}

The HIWIN MRG-P100 is an end-effector design robotic gait training system that allow patients to receive weightbearing gait re-education training without a suspension system. This non-suspension system offers a standing table and support modules for knees and pelvis, which allows patients to be in an anti-gravity upright position. The robotic system provides automated passive gait movements for the patients guided by the exoskeleton to allow repetitive stepping and balance training. The system provides programmable gait system with adjustable step length and walking speed for individual customization. More specifically, the system has three different pre-programmed gait trajectory modes (ellipse trajectory, flat ellipse trajectory and walking trajectory) with adjustable speed from $0.006-2.23 \mathrm{~km} /$ hr to allow best simulation of gait retraining pending on individual patient's presentation regarding affected muscle tone and spasticity of the gait muscle groups. Figure 1. is an illustration of the HIWIN Robotic Gait Training System (MRG-P100). 
(A)

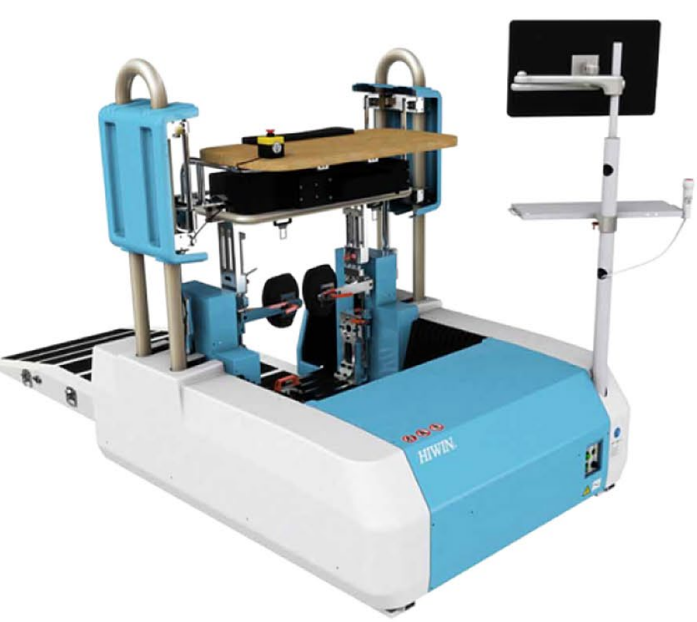

(B)

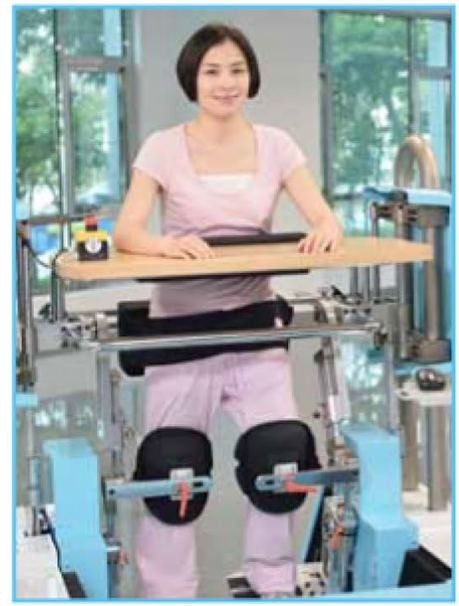

(C)

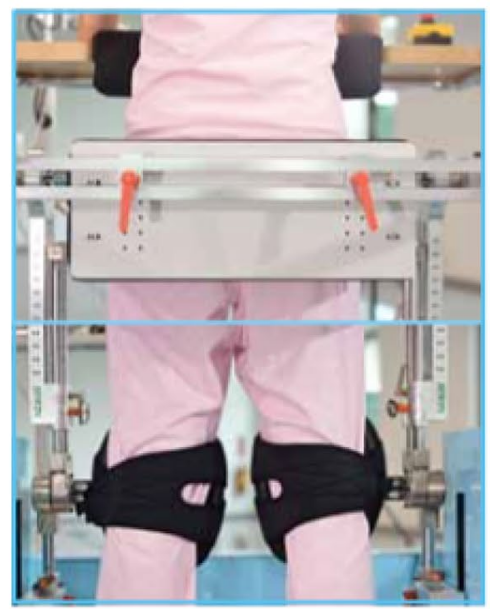

Fig. 1 a The HIWIN Robotic Gait Training System (MRG-P100); b illustrating patient supporting system with the trunk, pelvis and knee support from the front and $\mathbf{c}$ illustrating patient supporting system of the pelvis and knee from the back

\subsection{Procedure}

Eligibility for the study is assessed by the same hospital attending physician for all inpatient participants and when deemed appropriate, participant is then stratified through block sampling based on participant preference for either the standard hospital rehabilitation program only or with additional robotic gait retraining. Participant preference instead of randomization was used as the method of group allocation due to ethical consideration.

The standard hospital rehabilitation program for stroke comprises 45-60 min sessions daily for 3 weeks, utilizing hospital neurological physical therapy targeting positioning, balance, transfers, static standing table as well as gait mobility training. The robotic gait group received an additional 30minutes of robot-assisted gait training with the HIWIN MRG-P100 after each of their standard hospital rehabilitation session.

For the robot-assisted training program, an experienced physical therapist assisted the patient to transfer on to the robotic device via the built-in patient transfer system (Fig. 2). The therapist then adjusts the walking speed, step length as well as gait pattern in real time with the overarching aim of achieving the maximum progression of the gait settings to simulate a normal gait pattern that is tolerable by the patient. Patient's status of vital signs and perceived effort are closely monitored during the whole training period to ensure patient remain within a medically stable status that is pre-determined by their physician in charge. Only certified rehabilitation therapist that have completed the appropriate training with the operation of the HIWIN MRG-P100 is allowed to operate the gait training system. Participants

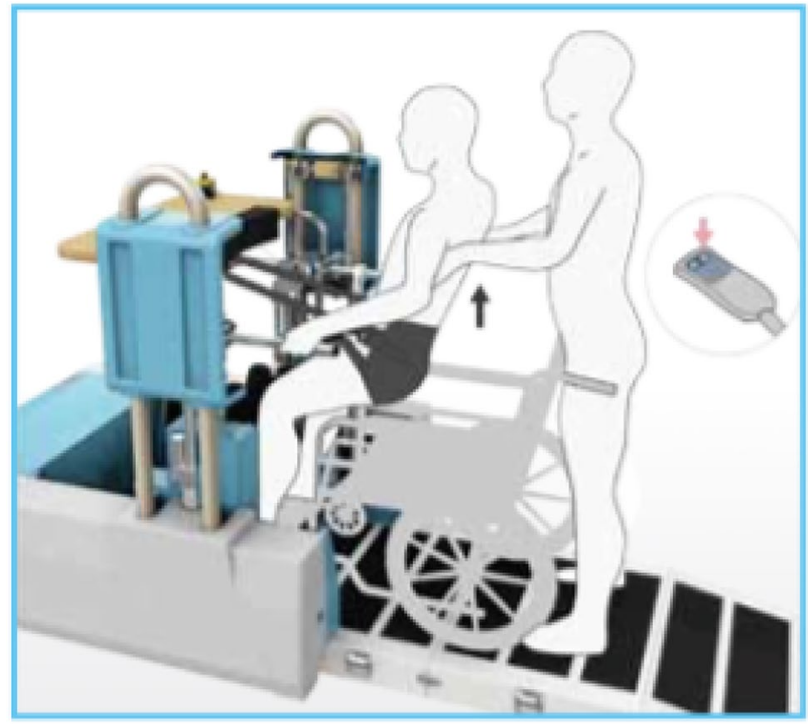

Fig. 2 The HIWIN Robotic Gait Training System (MRG-P100)'s patient transfer system

training parameters as well as vital signs were recorded during each training session.

\subsection{Outcome measures}

The following outcome measures were assessed pre-treatment and then after every five training sessions by the same chief resident of the department: 1. Berg Balance Scale (BBS) [20]; 2. Brunnstrom Stage [21]; 3. Pittsburgh Sleep Quality Index (PSQI)[22] and 4. Taiwanese Depression Questionnaire (TDQ) [23]. Overall, four assessment points 
were conducted for each participant and used for analysis. These outcome measures were chosen as they are the most commonly used clinical assessment tools for disability levels and psychological status post-stroke [24].

\subsubsection{Berg Balance Scale (BBS)}

The Berg Balance Scale (BBS) was initially developed to measure balance among older people with impairment in balance function by assessing performance of specific functional tasks. It is a 14-item list with each item consisting of a 5-point ordinal scale ranging from $0-4$, with 0 indicating the lowest level of function and 4 the highest level of function. Generally, the scores can be indicative of the following: 0-20: High fall risk; 21-40: Medium fall risk; 41-56: Low fall risk. It does not include the assessment of gait.

\subsubsection{Brunnstrom Stage}

The seven Brunnstrom stages were developed in the 1960s as a framework to understand and document how muscle control can be restored after stroke. Brunnstrom approach views spasticity and involuntary muscle movement not simply as symptoms but rather as part of the recovery process which can be used to direct treatment approach to aid recovery. The seven stages are defined as following: Stage 1: Flaccidity; Stage 2: Spasticity appears; Stage 3: Spasticity increases; Stage 4: Spasticity decreases; Stage 5: Complex movement returns; Stage 6: Spasticity disappears and Stage7: Normal function returns.

\subsubsection{Pittsburgh Sleep Quality Index (PSQI)}

The PSQI is a self-rated questionnaire which assess quality and pattern of sleep in the older adult. The questionnaire contains 19 individual items which generates seven "component" scores: 1. Subjective sleep quality; 2. Sleep latency; 3. Sleep duration; 4. Habitual sleep efficiency; 5. Sleep disturbances; 6. Use of sleeping medication and 7. Daytime dysfunction. Scoring is based on a 0 to 3 interval scale where 3 reflects the negative extreme. The global PSQI score is calculated by totalling the seven component scores and a global sum of 5 or greater score indicates a "poor" sleeper.

\subsubsection{Taiwanese Depression Questionnaire (TDQ)}

Different cultures need different methods for screening for depression or assessment of depression and TDQ is a culturally relevant self-report questionnaire commonly used to screen and assess depressive characteristics among Taiwanese. The TDQ consisted of three dimensions, with a total of 18 items on a 0 to 3 interval rating scale ranging from "less than 1 day per week" ( 0 ) to " 5 to 7 days per week" (3). The total score range is from 0 to 54 . A total of $<8$ points is considered to be emotionally stable; score of 9-14 points indicates slight depression; $15-18$ points indicates mild depression; $19-28$ points indicates moderate depression and a score of $>29$ points is indicative of severe depression.

\subsection{Statistical Analyses}

Statistical analyses were conducted using PASW Statistics (Version 20.0 for Mac, SPSS Inc., Chicago, IL, USA). Descriptive data are firstly extracted for all demographic parameters and where appropriate, a mix-design ANOVA were used to compare both within group (pre- and posttreatment) and between groups (with vs without robotassisted training) for all outcome measures. For categorical dependent variables where mix-design ANOVA isn't applicable, the Wilcoxon test was used to determine within group differences and the Mann-Whitney U-test for between group differences. A $p$ value of $\leq 0.05$ is considered to be statistically significant.

\section{Results}

\subsection{Participants}

Ninety participants were enrolled in the current study with thirty participants receiving just the standard hospital rehabilitation and sixty participants allocated to receive the additional daily robotic training. The group allocation and basic demographic details are provided in Fig. 3.

All ninety participants completed the pre-treatment assessment, however, nine participants due to administration reasons were either discharged or transferred to another hospital before the end of the study period and could not complete all three follow up assessments. One participant withdrew from the study due to personal reasons. Thus, complete assessment data were available for fifty-four participants in the robotic group and twenty-six for the control group. The breakdown of the dropouts is presented in Fig. 4.

Of the fifty-four participants in the robotic training group, twenty-six were ischemic stroke and twenty-eight were haemorrhagic stroke. The control group consisted of fourteen ischemic and twelve haemorrhagic stroke types. No significant differences were found $(p>0.05)$ for any of the demographic parameters between groups (Table 1). 
Fig. 3 Overall protocol and relevant participant group allocation and demographic details

\section{Recruitment:} and Rehabilitation at China Medical University Hospital
Department of Physical Medicine

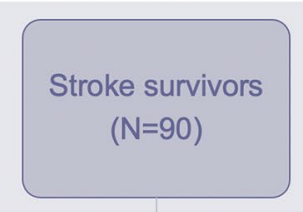

\begin{tabular}{|c|c|c|}
\hline $\begin{array}{l}\text { Intervention: } \\
\text { All patients received hospital based } \\
\text { rehabilitation everyday for } 3 \text { weeks, } \\
\text { with the experimental group } \\
\text { receiving a further } 30 \text { minutes of } \\
\text { robotic-assisted gait training }\end{array}$ & $\begin{array}{l}\text { Standard Hospital } \\
\text { Based } \\
\text { Rehabilitation } \\
(\mathrm{N}=30)\end{array}$ & $\begin{array}{l}\text { Standard Hospital } \\
\text { Based } \\
\text { Rehabilitation + } \\
\text { Robotic-assisted } \\
\text { Rehabilitation } \\
(\mathrm{N}=60) \\
\end{array}$ \\
\hline $\begin{array}{l}\text { Outcome: } \\
\text { - Berg balance scale } \\
\text { - Brunnstrom stage } \\
\text { - Pittsburgh Sleep Quality Index } \\
\text { Taiwanese Depression } \\
\text { Questionnaire }\end{array}$ & $\begin{array}{c}13 \text { male and } 17 \\
\text { females } \\
\text { mean age: } \\
58.2 \pm 12.6 \text { years }\end{array}$ & $\begin{array}{c}36 \text { males and } 24 \\
\text { females } \\
\text { mean age: } \\
57.8 \pm 13.1 \text { years }\end{array}$ \\
\hline
\end{tabular}
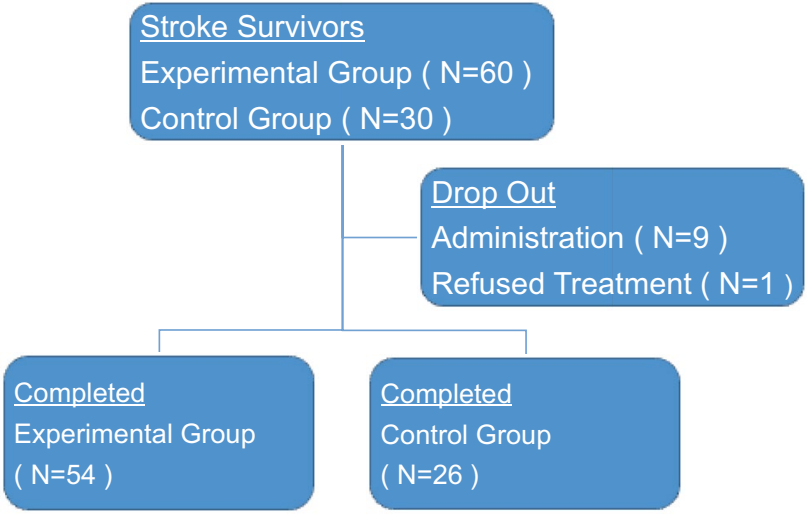

Fig. 4 Breakdown of the dropouts and the resultant participants numbers for each group

\subsection{Berg Balance Scale (BBS)}

The pre-training BBS score for the robotic training group was $15.1 \pm 3.2$ points and improved by 13.9 points to be $29.0 \pm 4.1$ points by the third assessment. The control group's pre-training BBS score was $18.8 \pm 3.7$ and improved by 9.7 points to be $28.5 \pm 4.8$ by the third assessment. Both groups demonstrated statistically significant within group improvement pre- vs post-training (robotic group $p=0.023$; control group $p=0.033$ ) and the post-hoc test showed a significant difference between the pre-training and all other assessment points for both groups. However, no significant difference $(p>0.1)$ was found between the two groups at any of the assessment points. Results of the change of BBS scores overtime are shown in Table 2.

In terms of points of improvement, for the robotic training group, $35 / 54(65 \%)$ improved at least 8 points compare to $14 / 26(54 \%)$ in the control group. The robotic group also had 24 (45\%) participants that improved more than 17 points with 3 participants improving more than 33 points. The control group had 5 (17\%) participants that improved more than 17 points on the BBS. Categorical improvement of scores are presented in Table 3.

\subsection{Brunnstrom Stage (Lower Limb)}

For the robotic training group, 30/54 (56\%) were classified as either Stage 2 or 3 pre-training and by the end of the training period, 34/54 (63\%) have improved to either stage 4 or 5. For the control group, most participants were classified
Table 1 Demographic and severity parameters of the two groups at baseline

\begin{tabular}{lllllcc}
\hline & Age & Gender (Male) & Onset of stroke (days) & BBS & PSQI & TDQ \\
\hline $\begin{array}{r}\text { Robotic training } \\
\text { group }(\mathrm{N}=54)\end{array}$ & $58.0 \pm 13.6$ & 36 & $11.3 \pm 2.1$ & $15.1 \pm 3.2$ & 9.5 & 15.9 \\
Control $(\mathrm{N}=26)$ & $57.9 \pm 12.8$ & 13 & $13.1 \pm 1.6$ & $18.8 \pm 3.7$ & 10.9 & 15.5 \\
Robotic vs Control & $\mathrm{p}=0.55$ & $\mathrm{p}=0.27$ & $\mathrm{p}=0.58$ & $\mathrm{p}=0.17$ & $\mathrm{p}=0.33$ & $\mathrm{p}=0.77$ \\
\hline
\end{tabular}

Robotic vs control: comparison of the demographic (age, gender, onset of stroke) and quantitative outcome scores (Berg Balance Scale, Pittsburgh Sleep Quality Index, Taiwanese Depression Questionnaire) at baseline between the two groups 
Table 2 Change of BBS scores overtime at different assessment points for the two groups

\begin{tabular}{|c|c|c|c|c|c|}
\hline & Pre-training & 1st Assessment & 2nd Asessment & 3rd Assessment & $\begin{array}{l}\text { Within } \\
\text { group } \\
\text { assessment }\end{array}$ \\
\hline $\begin{array}{r}\text { Robotic training } \\
\text { group }(\mathrm{N}=54)\end{array}$ & $15.1 \pm 3.2$ & $19.9 \pm 3.6$ & $25.2 \pm 4.8$ & $29.0 \pm 4.1$ & $\mathbf{p}=0.023 *$ \\
\hline Control $(\mathrm{N}=26)$ & $18.8 \pm 3.7$ & $21.7 \pm 2.4$ & $24.9 \pm 3.3$ & $28.5 \pm 4.8$ & $\mathbf{p}=\mathbf{0 . 0 3 3}^{*}$ \\
\hline Robotic vs Control & $\mathrm{p}=0.17$ & $\mathrm{p}=0.38$ & $\mathrm{p}=0.42$ & $\mathrm{p}=0.55$ & \\
\hline
\end{tabular}

Within group assessment: comparison of the change of BBS scores overtime within the group; Robotic vs control: comparison of the BBS scores at different assessment points between the two groups

Bold indicates $\mathrm{p}<0.05$
Table 3 Comparison of the categorical improvements of Berg Balance Score between the two groups

\begin{tabular}{lll}
\hline BBS score improvement & $\begin{array}{l}\text { Robotic training } \\
\text { group }(\mathrm{N}=54)\end{array}$ & Control $(\mathrm{N}=26)$ \\
\hline$<8$ points & $19(35 \%)$ & $12(46 \%)$ \\
$8-16$ points & $11(20 \%)$ & $9(35 \%)$ \\
$17-24$ points & $13(24 \%)$ & $2(8 \%)$ \\
$25-32$ points & $8(15 \%)$ & $3(12 \%)$ \\
$>33$ points & $3(6 \%)$ & $0(0 \%)$ \\
\hline
\end{tabular}

BBS Berg Balance Scale; BBS Score improvement: Change of BBS points from pre-treatment to last assessment

Table 4 Comparison of the Brunnstrom stage (lower limb) improvements between the two groups, pre- vs post-treatment

\begin{tabular}{lll}
\hline $\begin{array}{l}\text { Brunnstrom stage } \\
\text { improvement }\end{array}$ & $\begin{array}{l}\text { Robotic training group } \\
(\mathrm{N}=54)\end{array}$ & Control (N=26) \\
\hline Unchanged & $20(37 \%)$ & $14(54 \%)$ \\
1 stage & $29(54 \%)$ & $9(35 \%)$ \\
2 stages & $3(6 \%)$ & $3(11 \%)$ \\
3 stages & $2(4 \%)$ & $0(0 \%)$ \\
\hline
\end{tabular}

Brunnstrom stage improvement: Change of Brunnstrom stage from pre-treatment to last assessment point

as either stage 4 or $5(18 / 26)$ and remained till the end of the assessment point $(21 / 26)$.

In terms of change of stage pre- and post-training, for the robotic training group, 29/54 (54\%) participants improved by one stage, 3 participants improved by two stages and one participant improved from stage two to stage five. Only 20/54 (37\%) did not show improvement in Brunnstrom stage. For the control group, 14/26 (54\%) participants remain unchanged of the Brunnstrom stage post treatment and nine (35\%) improved by one stage and three $(12 \%)$ participants improved by two stages. A summary of the Brunnstrom stage improvements are summarized in Table 4.

\subsection{Pittsburgh Sleep Quality Index (PSQI)}

For the robotic training group, the mean pre-treatment score was 9.5 and was 8.0 at the last assessment point. The pretreatment score was 10.9 and improved to 9.3 for the control group. No statistically significant difference was found for both within and between group comparisons $(\mathrm{p}>0.4)$. The PSQI results are summarized in Table 5.

\subsection{Taiwanese Depression Questionnaire}

For the robotic training group, the mean pre-treatment score was 15.9 and improved by 3.6 points with an end score of 12.3. The pre-treatment score was 15.5 and improved by 2.1 point to 13.4 for the control group. No statistically significant difference was found for both within and between group comparisons ( $p>0.4)$. The Taiwanese Depression Questionnaire results are summarized in Table 6.
Table 5 Change of Pittsburgh Sleep Quality Index (PSQI) scores overtime at different assessment points for the two groups

\begin{tabular}{|c|c|c|c|c|c|}
\hline & Pre-treatment & 1 st assessment & 2nd asessment & 3rd assessment & $\begin{array}{l}\text { Within } \\
\text { group com- } \\
\text { parison }\end{array}$ \\
\hline $\begin{array}{l}\text { Robotic training } \\
\text { group }(\mathrm{N}=54)\end{array}$ & 9.5 & 9.1 & 8.5 & 8 & $\mathrm{p}=0.48$ \\
\hline Control $(\mathrm{N}=26)$ & 10.9 & 10 & 9.3 & 9.3 & $\mathrm{p}=0.32$ \\
\hline Robotic vs Control & $\mathrm{p}=0.33$ & $\mathrm{p}=0.41$ & $\mathrm{p}=0.56$ & $\mathrm{p}=0.37$ & \\
\hline
\end{tabular}

Within group assessment: comparison of the change of PSQI scores overtime within the group; Robotic vs control: comparison of the PSQI scores at different assessment points between the two groups 
Table 6 Change of Taiwanese Depression Questionnaire (TDQ) scores overtime at different assessment points for the two groups

\begin{tabular}{|c|c|c|c|c|c|}
\hline & Pre-treatment & 1 st assessment & 2nd asessment & 3rd assessment & $\begin{array}{l}\text { Within } \\
\text { group com- } \\
\text { parison }\end{array}$ \\
\hline $\begin{array}{r}\text { Robotic training } \\
\text { group }(\mathrm{N}=54)\end{array}$ & 15.9 & 14.2 & 12.6 & 12.3 & $\mathrm{p}=0.55$ \\
\hline Control $(\mathrm{N}=26)$ & 15.5 & 15.3 & 13.2 & 13.4 & $\mathrm{p}=0.44$ \\
\hline Robotic vs Control & $\mathrm{p}=0.77$ & $\mathrm{p}=0.58$ & $\mathrm{p}=0.43$ & $\mathrm{p}=0.48$ & \\
\hline
\end{tabular}

Within group assessment: comparison of the change of TDQ scores overtime within the group; Robotic vs control: comparison of the TDQ scores at different assessment points between the two groups

\section{Discussion}

In this study, we attempted to assess the physical and psychological benefits of additional robotic gait training for acute stroke patients when compared to standard hospital neurorehabilitation alone. Our results supported our hypotheses that although both groups demonstrated statistically comparable improvements over the study period based on the selected outcome measures, clinical benefits of the additional robot-assisted gait training were observed for the functional improvement of the patients.

Berg balance scale (BBS) is one of the most commonly used objective measure that assesses static balance and fall risks in stroke patients. Our results indicated that statistically significant improvement was observed for both groups and the robotic training group was not statistically better than the control group by the end of the study period. This is consistent with the findings of a 2014 systematic review [25] where the authors found significant improvements in balance scores measured with the BBS after robot-assisted gait training but no significant difference between the intervention and control groups were found. However, it was suggested that a lack of statistically significant difference should not be directly assumed to be of no clinical difference.

As proposed by Stevenson and colleagues, [26] the minimum detectable change score (MDC) in acute stroke patients for BBS is at least 8 points. Current study indicated that a greater proportion of the robotic training participant $(65 \%$ vs $54 \%$ ) achieved this clinically significant change. Moreover, $45 \%$ of the robotic training group achieved $2 \times \mathrm{MDC}$ improvement of greater than 17 points where only $17 \%$ of the control group demonstrated such improvement. Such observation is consistent with a more recent systematic review [27] where the meta-analysis of thirty-one randomized controlled trails revealed robot-assisted therapy produced an increase in the BBS score compared with stroke patients without the robotic training. Many different mechanisms have been proposed to explain how robotassisted gait training system can improve balance function in stroke patients but we speculate that the tilt-table design of the MRG-P100 system provided adequate weight bearing support for patients which alleviate their fear of falls and subsequently regain the confidence in participating balancerelated locomotion practice.

Comparable to BBS results, Brunnstrom stage results also demonstrated a similar pattern where both groups demonstrated comparable improvements post-treatment. However, a closer assessment of the changes in Brunnstrom stages comparing the percentage changes at the last assessment point relative to pre-treatment indicated a greater number of participants in the robotic training group with at least one stage of change. However, more than half of the participants' s stage in the control group remain unchanged. This finding supports previous finding that robotic training combined with conventional physiotherapy produced better improvement in Brunnstrom stages than conventional physiotherapy alone [28]. However, it is also acknowledged that the spread of the Brunnstrom stages were different between the two groups per-training, with more of the control group classified as stage 4 which might have also contributed to the smaller improvement.

In contrast to BBS and Brunnstrom stage results, both the psychological parameters, Pittsburgh Sleep Quality Index (PSQI) and Taiwanese Depression Questionnaires (TDQ) did not show any significant within and between group improvements over the study period. This is somewhat surprising given most of the available literature indicates that the integration of technology in stroke rehabilitation often lead to improvement of quality of life and psychological status.[29-31] Such discrepancies of the current study results may be due to the fact that our sample consisted of acute ( $<3$ month) stroke patients where most available literature have looked at the change of psychological status in chronic stage of stroke. It is speculated that during the acute stage of stroke recovery, the self-reflected psychological status may be more strongly associated with disease and recovery rate and the different types of intervention appears to have less contribution.

It is acknowledged that the lack of statistically significant difference for most outcome measures between the those receiving additional robotic training and those without might be contributed by the fact that both groups received the same standard hospital neurorehabilitation and the sole additional benefits of robotic training isn't 
adequate to achieve a statistical difference. In particular, the hospital neurorehabilitation utilized by the authors' institution incorporates and emphasize early and intense mobilization, transfer and balance training for acute stroke patients, which may further "wash-out" the benefits of robotic training. However, due to ethical consideration, the study design could not omit or reduce the standard in-hospital neurorehabilitation provided for all patients.

It must also be noted that as the first 3 months post-stroke are usually considered as the golden phase of spontaneous recovery and such improvement may inevitably reduce the observable benefit of robotic training. It is plausible that extending the intervention period for more than 3 weeks may better differentiate group differences, however, due to the policy restriction of our national health insurance system where patients are required to transfer to a different institution to continue with treatment after a maximum of 28 days at a single institution, it was logistically difficult to extend the intervention period. Nevertheless, it is acknowledged that the lack of statistically significant differences between groups could be a true reflection of the treatment efficacy of the end-effector robotic gait training design utilized in the study. In a recent review of robotic rehabilitation in stroke patients, it was revealed that electromechanical-assisted devices with exoskeleton design appear to reach better results than end-effector design [32]. The lack of functional outcome to determine lower limb ambulation or muscle tone capacity should also be considered as a limitation of the current study design and must be considered for further studies.

\section{Conclusion}

In conclusion, the addition of robotic gait training on top of standard hospital neurorehabilitation for acute stroke patients appear to produce a slightly greater improvement in clinical functional outcomes, which is not transferred to psychological status. Based on the available results, it is our recommendations that patients with limited ambulatory ability can consider incorporating robot-assisted gait training into the rehabilitation program, especially in clinical settings with scarce clinical resources to be a cost- and labor-efficient supplementary treatment strategy.

Author contributions Overall, AC and FC contributed equally as the first authors of this manuscript. AC contributed to the design of the study, analysis and interpretation of data and preparation of the initial draft of the manuscript. FC contributed to participant screening, data collection, analysis and interpretation as well as the preparation of the revised manuscript. $\mathrm{CH}$ contributed to data collection and data analysis. NM and PY contributed to the study design and review of the manuscript. LC contributed to the study design, analysis and interpretation of data and the final approval of the manuscript.
Funding This research was supported by Industry-University Cooperative Research Fund (104426DA and 10642605) between HIWIN Technologies Cooperation, China Medical University and China Medical University Hospital.

Data Availability The data generated and/or used for analysis that is associated with the preparation of this manuscript is available from the corresponding author.

Code Availability The code generated and/or used for analysis that is associated with the preparation of this manuscript is available from the corresponding author.

\section{Compliance with Ethical Standards}

Conflicts of interest HIWN Technologies Cooperation provided financial support for the study, however, has no input in the study design and subject matter or materials discussed in this manuscript. The authors have no other conflict of interest to disclose.

Ethical approval This study and its protocols were approved by the China Medical University Hospital Research Ethics Committee (CMUH105-REC1-037).

Consent to participate All participants provided written informed consent as per the Ethics approved protocol.

Open Access This article is licensed under a Creative Commons Attribution 4.0 International License, which permits use, sharing, adaptation, distribution and reproduction in any medium or format, as long as you give appropriate credit to the original author(s) and the source, provide a link to the Creative Commons licence, and indicate if changes were made. The images or other third party material in this article are included in the article's Creative Commons licence, unless indicated otherwise in a credit line to the material. If material is not included in the article's Creative Commons licence and your intended use is not permitted by statutory regulation or exceeds the permitted use, you will need to obtain permission directly from the copyright holder. To view a copy of this licence, visit http://creativecommons.org/licenses/by/4.0/.

\section{References}

1. Langhorne, P., Bernhardt, J., \& Kwakkel, G. (2011). Stroke rehabilitation. Lancet, 377(9778), 1693-1702. https://doi.org/10.1016/ s0140-6736(11)60325-5.

2. Jaracz, K., Grabowska-Fudala, B., Gorna, K., \& Kozubski, W. (2014). Consequences of stroke in the light of objective and subjective indices: a review of recent literature. Neurologia i Neurochirurgia Polska, 48(4), 280-286. https://doi.org/10.1016/j.pjnns .2014.07.004.

3. Barker-Collo, S., Feigin, V. L., Parag, V., Lawes, C. M., \& Senior, H. (2010). Auckland Stroke Outcomes Study Part 2 Cognition and functional outcomes 5 years poststroke. Neurology, 75(18), 1608-1616. https://doi.org/10.1212/WNL.0b013e3181fb44c8.

4. Park, G. Y., Im, S., Lee, S. J., \& Pae, C. U. (2016). The Association between Post-Stroke Depression and the Activities of Daily Living/Gait Balance in Patients with First-Onset Stroke Patients. Psychiatry Investig, 13(6), 659-664. https://doi.org/10.4306/ pi.2016.13.6.659.

5. Paolucci, S., Iosa, M., Coiro, P., Venturiero, V., Savo, A., De Angelis, D., et al. (2019). Post-stroke Depression Increases 
Disability More Than 15\% in Ischemic Stroke Survivors: A CaseControl Study. Front Neurol, 10, 926. https://doi.org/10.3389/ fneur.2019.00926.

6. Esquenazi, A., \& Packel, A. (2012). Robotic-assisted gait training and restoration. American Journal of Physical Medicine and Rehabilitation, 91(11), S217-227. https://doi.org/10.1097/ PHM.0b013e31826bce18.

7. Sivan, M., O'Connor, R. J., Makower, S., Levesley, M., \& Bhakta, B. (2011). Systematic review of outcome measures used in the evaluation of robot-assisted upper limb exercise in stroke. Journal of Rehabilitation Medicine, 43(3), 181-189. https://doi. org/10.2340/16501977-0674.

8. Mehrholz, J., Thomas, S., Werner, C., Kugler, J., Pohl, M., \& Elsner, B. (2017). Electromechanical-assisted training for walking after stroke. Cochrane Database Systematic Review, 5(5), Cd006185. https://doi.org/10.1002/14651858.CD006185.pub4.

9. Hidler, J., Nichols, D., Pelliccio, M., \& Brady, K. (2005). Advances in the understanding and treatment of stroke impairment using robotic devices. Top Stroke Rehabil, 12(2), 22-35. https://doi.org/10.1310/ryt5-62n4-ctvx-8jte.

10. Chang, W. H., \& Kim, Y. H. (2013). Robot-assisted Therapy in Stroke Rehabilitation. J Stroke, 15(3), 174-181. https://doi. org/10.5853/jos.2013.15.3.174.

11. Hidler, J., Nichols, D., Pelliccio, M., Brady, K., Campbell, D. D., Kahn, J. H., et al. (2009). Multicenter randomized clinical trial evaluating the effectiveness of the Lokomat in subacute stroke. Neurorehabil Neural Repair, 23(1), 5-13. https://doi. org/10.1177/1545968308326632.

12. Hornby, T. G., Campbell, D. D., Kahn, J. H., Demott, T., Moore, J. L., \& Roth, H. R. (2008). Enhanced gait-related improvements after therapist- versus robotic-assisted locomotor training in subjects with chronic stroke: a randomized controlled study. Stroke, 39(6), 1786-1792. https://doi.org/10.1161/strokeaha.107.504779.

13. Werner, C., Von Frankenberg, S., Treig, T., Konrad, M., \& Hesse, S. (2002). Treadmill training with partial body weight support and an electromechanical gait trainer for restoration of gait in subacute stroke patients: a randomized crossover study. Stroke, 33(12), 2895-2901.

14. Mayr, A., Kofler, M., Quirbach, E., Matzak, H., Frohlich, K., \& Saltuari, L. (2007). Prospective, blinded, randomized crossover study of gait rehabilitation in stroke patients using the Lokomat gait orthosis. Neurorehabil Neural Repair, 21(4), 307-314. https ://doi.org/10.1177/1545968307300697.

15. Husemann, B., Muller, F., Krewer, C., Heller, S., \& Koenig, E. (2007). Effects of locomotion training with assistance of a robotdriven gait orthosis in hemiparetic patients after stroke: a randomized controlled pilot study. Stroke, 38(2), 349-354. https://doi. org/10.1161/01.STR.0000254607.48765.cb.

16. Peurala, S. H., Tarkka, I. M., Pitkanen, K., \& Sivenius, J. (2005). The effectiveness of body weight-supported gait training and floor walking in patients with chronic stroke. Archives of Physical Medicine and Rehabilitation, 86(8), 1557-1564. https://doi. org/10.1016/j.apmr.2005.02.005.

17. Westlake, K. P., \& Patten, C. (2009). Pilot study of Lokomat versus manual-assisted treadmill training for locomotor recovery post-stroke. J Neuroeng Rehabil, 6, 18. https://doi. org/10.1186/1743-0003-6-18.

18. Lum, P., Reinkensmeyer, D., Mahoney, R., Rymer, W. Z., \& Burgar, C. (2002). Robotic devices for movement therapy after stroke: current status and challenges to clinical acceptance. Top Stroke Rehabil, 8(4), 40-53. https://doi. org/10.1310/9kfm-kf81-p9a4-5ww0.
19. H.-Y., J. . (2017). Rehabilitation in subacute and chronic stage after stroke. In S. H. Lee (Ed.), Stroke revisited: diagnosis and treatment of ischemic stroke (pp. 351-360). Singapore: Springer.

20. Berg, K. O., Wood-Dauphinee, S. L., Williams, J. I., \& Maki, B. (1992). Measuring balance in the elderly: validation of an instrument. Canadian Journal of Public Health, 83(Suppl 2), S7-11.

21. Brunnstrom, S. (1966). Motor testing procedures in hemiplegia: based on sequential recovery stages. Physical Therapy, 46(4), 357-375. https://doi.org/10.1093/ptj/46.4.357.

22. Buysse, D. J., Reynolds, C. F., 3rd., Monk, T. H., Berman, S. R., \& Kupfer, D. J. (1989). The Pittsburgh Sleep Quality Index: a new instrument for psychiatric practice and research. Psychiatry Research, 28(2), 193-213. https://doi.org/10.1016/01651781(89)90047-4.

23. Lee, Y., Yang, M. J., Lai, T. J., Chiu, N. M., \& Chau, T. T. (2000). Development of the Taiwanese Depression Questionnaire. Chang Gung Med J, 23(11), 688-694.

24. Burton, L., \& Tyson, S. F. (2015). Screening for cognitive impairment after stroke: A systematic review of psychometric properties and clinical utility. Journal of Rehabilitation Medicine, 47(3), 193-203. https://doi.org/10.2340/16501977-1930.

25. Swinnen, E., Beckwée, D., Meeusen, R., Baeyens, J. P., \& Kerckhofs, E. (2014). Does robot-assisted gait rehabilitation improve balance in stroke patients? A systematic review. Top Stroke Rehabil, 21(2), 87-100. https://doi.org/10.1310/tsr2102-87.

26. Stevenson, T. J. (2001). Detecting change in patients with stroke using the Berg Balance Scale. Aust J Physiother, 47(1), 29-38. https://doi.org/10.1016/s0004-9514(14)60296-8.

27. Zheng, Q. X., Ge, L., Wang, C. C., Ma, Q. S., Liao, Y. T., Huang, P. P., et al. (2019). Robot-assisted therapy for balance function rehabilitation after stroke: A systematic review and meta-analysis. International Journal of Nursing Studies, 95, 7-18. https://doi. org/10.1016/j.ijnurstu.2019.03.015.

28. Dundar, U., Toktas, H., Solak, O., Ulasli, A. M., \& Eroglu, S. (2014). A comparative study of conventional physiotherapy versus robotic training combined with physiotherapy in patients with stroke. Top Stroke Rehabil, 21(6), 453-461. https://doi. org/10.1310/tsr2106-453.

29. Linder, S. M., Rosenfeldt, A. B., Bay, R. C., Sahu, K., Wolf, S. L., \& Alberts, J. L. (2015). Improving Quality of Life and Depression After Stroke Through Telerehabilitation. American Journal of Occupational Therapy. https://doi.org/10.5014/ajot.2015.01449 8.

30. Calabrò, R. S., De Cola, M. C., Leo, A., Reitano, S., Balletta, T., Trombetta, G., et al. (2015). Robotic neurorehabilitation in patients with chronic stroke: psychological well-being beyond motor improvement. International Journal of Rehabilitation Research, 38(3), 219-225. https://doi.org/10.1097/mrr.00000 00000000114.

31. Trompetto, C., Marinelli, L., Mori, L., Cossu, E., Zilioli, R., Simonini, M., et al. (2013). Postactivation depression changes after robotic-assisted gait training in hemiplegic stroke patients. Gait Posture, 38(4), 729-733. https://doi.org/10.1016/j.gaitp ost.2013.03.011.

32. Bruni, M. F., Melegari, C., De Cola, M. C., Bramanti, A., Bramanti, P., \& Calabrò, R. S. (2018). What does best evidence tell us about robotic gait rehabilitation in stroke patients: A systematic review and meta-analysis. J Clin Neurosci, 48, 11-17. https://doi. org/10.1016/j.jocn.2017.10.048. 